\title{
COMPUTATION OF MATRIX CHAIN PRODUCTS. PART I*
}

\author{
T. C. HU† AND M. T. SHING $\dagger$
}

\begin{abstract}
This paper considers the computation of matrix chain products of the form $M_{1} \times M_{2} \times \cdots \times$ $M_{n-1}$. If the matrices are of different dimensions, the order in which the product is computed affects the number of operations. An optimum order is an order which minimizes the total number of operations. We present some theorems about an optimum order of computing the matrices. Based on these theorems, an $O(n \log n)$ algorithm for finding an optimum order will be presented in Part II.
\end{abstract}

Key words. matrix multiplication, polygon partition, dynamic programming

1. Introduction. Consider the evaluation of the product of $n-1$ matrices

$$
M=M_{1} \times M_{2} \times \cdots \times M_{n-1},
$$

where $M_{i}$ is a $w_{i} \times w_{i+1}$ matrix. Since matrix multiplication satisfies the associative law, the final result $M$ in (1) is the same for all orders of multiplying the matrices. However, the order of multiplication greatly affects the total number of operations to evaluate $M$. The problem is to find an optimum order of multiplying the matrices such that the total number of operations is minimized. Here, we assume that the number of operations to multiply a $p \times q$ matrix by a $q \times r$ matrix is $p q r$.

In [1], [7], a dynamic programming algorithm is used to find an optimum order. The algorithm needs $O\left(n^{3}\right)$ time and $O\left(n^{2}\right)$ space. In [2], Chandra proposed a heuristic algorithm to find an order of computation which requires no more than $2 T_{o}$ operations where $T_{o}$ is the total number of operations to evaluate (1) in an optimum order. This heuristic algorithm needs only $O(n)$ time. Chin [3] proposed an improved heuristic algorithm to give an order of computation which requires no more than $1.25 T_{o}$. This improved heuristic algorithm also needs only $O(n)$ time.

In this paper we first transform the matrix chain product problem into a problem in graph theory-the problem of partitioning a convex polygon into nonintersecting triangles, see [9], [10], [11], [12]; then we state several theorems about the optimum partitioning problem. Based on these theorems, an $O(n \log n)$ algorithm for finding an optimum partition is developed.

2. Partitioning a convex polygon. Given an $n$-sided convex polygon, such as the hexagon shown in Fig. 1, the number of ways to partition the polygon into $(n-2)$ triangles by nonintersecting diagonals is the Catalan number (see for example, Gould [8]). Thus, there are 2 ways to partition a convex quadrilateral, 5 ways to partition a convex pentagon, and 14 ways to partition a convex hexagon.

Let every vertex $V_{i}$ of the polygon have a positive weight $w_{i}$. We can define the cost of a given partition as follows: The cost of a triangle is the product of the weights of the three vertices, and the cost of partitioning a polygon is the sum of the costs of all its triangles. For example, the cost of the partition of the hexagon in Fig. 1 is

$$
w_{1} w_{2} w_{3}+w_{1} w_{3} w_{6}+w_{3} w_{4} w_{6}+w_{4} w_{5} w_{6}
$$

\footnotetext{
* Received by the editors May 19, 1980, and in final revised form September 9, 1981. This research was supported in part by the National Science Foundation under grant MCS-80-03362 and by the U.S. Army Research Office under grant DAAG29-80-C-0029.

$\uparrow$ Department of Electrical Engineering and Computer Sciences, University of California, San Diego, California 92093.
} 


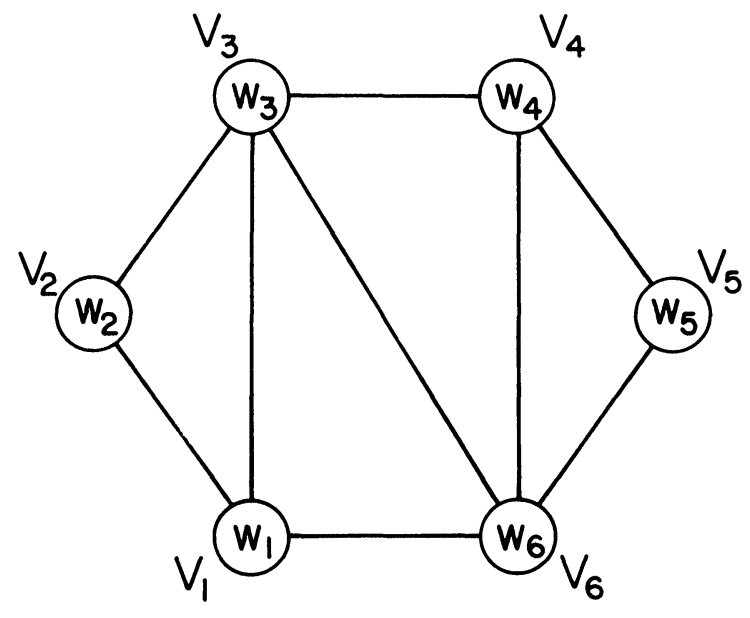

FIG. 1

If we erase the diagonal from $V_{3}$ to $V_{6}$ and replace it by the diagonal from $V_{1}$ to $V_{4}$, then the cost of the new partition will be

$$
w_{1} w_{2} w_{3}+w_{1} w_{3} w_{4}+w_{1} w_{4} w_{6}+w_{4} w_{5} w_{6} .
$$

We will prove that an order of multiplying $n-1$ matrices corresponds to a partition of a convex polygon with $n$ sides. The cost of the partition is the total number of operations needed in multiplying the matrices. For brevity, we shall use $n$-gon to mean a convex polygon with $n$ sides, and the partition of an $n$-gon to mean the partitioning of an n-gon into $n-2$ nonintersecting triangles.

For any $n$-gon, one side of the $n$-gon will be considered to be its base, and will usually be drawn horizontally at the bottom such as the side $V_{1}-V_{6}$ in Fig. 1 . This side will be called the base; all other sides are considered in a clockwise way. Thus, $V_{1}-V_{2}$ is the first side, $V_{2}-V_{3}$ the second side, $\cdots$ and $V_{5}-V_{6}$ the fifth side.

The first side represents the first matrix in the matrix chain and the base represents the final result $M$ in (1). The dimensions of a matrix are the two weights associated with the two end vertices of the side. Since the adjacent matrices are compatible, the dimensions $w_{1} \times w_{2}, w_{2} \times w_{3}, \cdots, w_{n-1} \times w_{n}$ can be written inside the vertices as $w_{1}, w_{2}, \cdots, w_{n}$. The diagonals are the partial products. A partition of an $n$-gon corresponds to an alphabetic tree of $n-1$ leaves or the parenthesis problem of $n-1$ symbols (see, for example, Gardner [6]). It is easy to see the one-to-one correspondence between the multiplication of $n-1$ matrices to either the alphabetic binary tree or the parenthesis problem of $n-1$ symbols. Here, we establish the correspondence between the matrix-chain product and the partition of a convex polygon directly.

LEMMA 1. Any order of multiplying $n-1$ matrices corresponds to a partition of an n-gon.

Proof. We shall use induction on the number of matrices. For two matrices of dimensions $w_{1} \times w_{2}, w_{2} \times w_{3}$, there is only one way of multiplication; this corresponds to a triangle where no further partition is required. The total number of operations in multiplication is $w_{1} w_{2} w_{3}$, the product of the three weights of the vertices. The resulting matrix has dimension $w_{1} \times w_{3}$. For three matrices, the two orders of multiplication $\left(M_{1} \times M_{2}\right) \times M_{3}$ and $M_{1} \times\left(M_{2} \times M_{3}\right)$ correspond to the two ways of partitioning a 4 -gon. Assume that this lemma is true for $k$ matrices where $k \leqq n-2$, and we now consider $n-1$ matrices. The $n$-gon is shown in Fig. 2. 


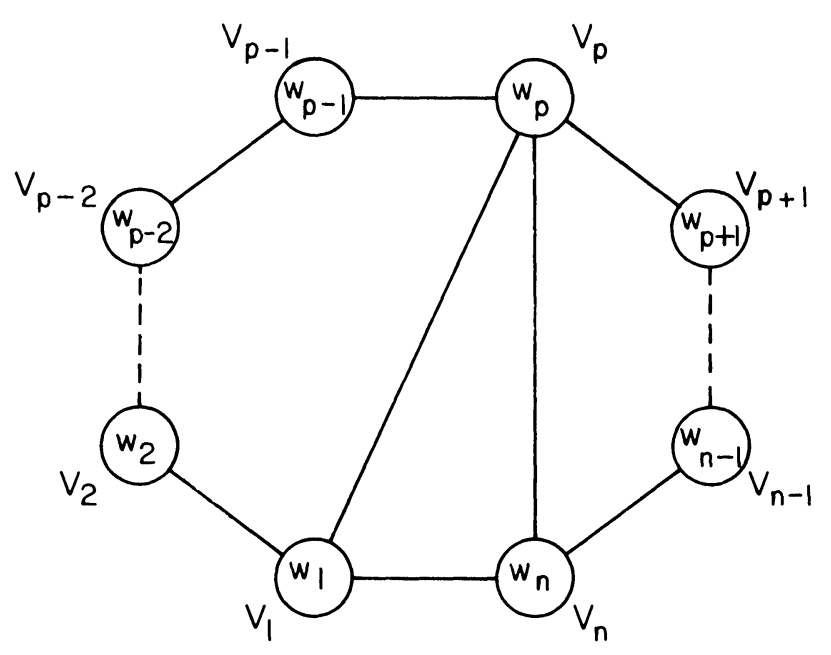

FIG. 2

Let the order of multiplication be represented by

$$
M=\left(M_{1} \times M_{2} \times \cdots \times M_{p-1}\right) \times\left(M_{p} \times \cdots \times M_{n-1}\right) ;
$$

i.e., the final matrix is obtained by multiplying a matrix of dimension $\left(w_{1} \times w_{p}\right)$ and a matrix of dimension $\left(w_{p} \times w_{n}\right)$. Then in the partition of the $n$-gon, we let the triangle with vertices $V_{1}$ and $V_{n}$ have the third vertex $V_{p}$. The polygon $V_{1}-V_{2}-\cdots-V_{p}$ is a convex polygon of $p$ sides with base $V_{1}-V_{p}$ and its partition corresponds to an order of multiplying matrices $M_{1}, \cdots, M_{p-1}$, giving a matrix of dimension $w_{1} \times w_{p}$. Similarly, the partition of the polygon $V_{p}-V_{p+1}-\cdots-V_{n}$ with base $V_{p}-V_{n}$ corresponds to an order of multiplying matrices $M_{p}, \cdots, M_{n-1}$, giving a matrix of dimension $w_{p} \times w_{n}$. Hence the triangle $V_{1} V_{p} V_{n}$ with base $V_{1}-V_{n}$ represents the multiplication of the two partial products, giving the final matrix of dimension $w_{1} \times w_{n}$.

LEMMA 2. The minimum numbers of operations needed to evaluate the following matrix chain products are identical.

$$
\begin{gathered}
M_{1} \times M_{2} \times \cdots \times M_{n-2} \times M_{n-1}, \\
M_{n} \times M_{1} \times \cdots \times M_{n-3} \times M_{n-2}, \\
\vdots \\
M_{2} \times M_{3} \times \cdots \times M_{n-1} \times M_{n},
\end{gathered}
$$

where $M_{i}$ has dimension $w_{i} \times w_{i+1}$ and $w_{n+1} \equiv w_{1}$. Note that in the first matrix chain, the resulting matrix is of dimension $w_{1} \times w_{n}$. In the last matrix chain, the resulting matrix is of dimension $w_{2} \times w_{1}$. But in all the cases, the total number of operations in the optimum orders of multiplication is the same.

Proof. The cyclic permutations of the $n-1$ matrices all correspond to the same $n$-gon and thus have the same optimum partitions.

(This lemma was obtained independently in [4] with a long proof.)

From now on, we shall concentrate only on the partitioning problem.

The diagonals inside the polygon are called arcs. Thus, one easily verifies inductively that every partition consists of $n-2$ triangles formed by $n-3$ arcs and $n$ sides.

In a partition of an $n$-gon, the degree of a vertex is the number of arcs incident on the vertex plus two (since there are two sides incident on every vertex). 
LEMMA 3. In any partition of an $n$-gon, $n \geqq 4$, there are at least two triangles, each having a vertex of degree two. (For example, in Fig. 1, the triangle $V_{1} V_{2} V_{3}$ has vertex $V_{2}$ with degree 2 and the triangle $V_{4} V_{5} V_{6}$ has vertex $V_{5}$ with degree 2.) (See also [5].)

Proof. In any partition of an $n$-gon, there are $n-2$ nonintersecting triangles formed by $n-3$ arcs and $n$ sides. And for any $n \geqq 4$, no triangle can be formed by 3 sides. Let $x$ be the number of triangles with two sides and one arc, $y$ be the number of triangles with one side and two arcs, and $z$ be the number of triangles with three arcs. Since an arc is used in two triangles, we have

$$
x+2 y+3 z=2(n-3) .
$$

Since the polygon has $n$ sides, we have

$$
2 x+y=n .
$$

From (4) and (5), we get

$$
3 x=3 z+6 .
$$

Since $z \geqq 0$, we have $x \geqq 2$.

LEMMA 4. Let $P$ and $P^{\prime}$ both be $n$-gons where the corresponding weights of the vertices satisfy $w_{i} \leqq w_{i}^{\prime}$. Then the cost of an optimum partition of $P$ is less than or equal to the cost of an optimum partition of $P^{\prime}$.

Proof. Omitted.

If we use $C\left(w_{1}, w_{2}, w_{3}, \cdots, w_{k}\right)$ to mean the minimum cost of partitioning the $k$-gon with weights $w_{i}$ optimally, Lemma 4 can be stated as

$$
C\left(w_{1}, w_{2}, \cdots, w_{k}\right) \leqq C\left(w_{1}^{\prime}, w_{2}^{\prime}, \cdots, w_{k}^{\prime}\right) \text { if } w_{i} \leqq w_{i}^{\prime} .
$$

We say that two vertices are connected in an optimum partition if the two vertices are connected by an arc or if the two vertices are adjacent to the same side.

In the rest of the paper, we shall use $V_{1}, V_{2}, \cdots, V_{n}$ to denote vertices which are ordered according to their weights, i.e., $w_{1} \leqq w_{2} \leqq \cdots \leqq w_{n}$. To facilitate the presentation, we introduce a tie-breaking rule for vertices of equal weights.

If there are two or more vertices with weights equal to the smallest weight $w_{1}$, we can arbitrarily choose one of these vertices to be the vertex $V_{1}$. Once the vertex $V_{1}$ is chosen, further ties in equal weights are resolved by regarding the vertex which is closer to $V_{1}$ in the clockwise direction to be of less weight. With this tie-breaking rule, we can unambiguously label the vertices $V_{1}, V_{2}, \cdots, V_{n}$ for each choice of $V_{1}$. A vertex $V_{i}$ is said to be smaller than another vertex $V_{j}$, denoted by $V_{i}<V_{i}$, either if $w_{i}<w_{j}$ or if $w_{i}=w_{j}$ and $i<j$. We say that $V_{i}$ is the smallest vertex in a subpolygon if it is smaller than any other vertices in the subpolygon.

After the vertices are labeled, we define an arc $V_{i}-V_{j}$ to be less than another $\operatorname{arc} V_{p}-V_{q}$ if

$$
\min (i, j)<\min (p, q) \text { or }\left\{\begin{array}{l}
\min (i, j)=\min (p, q), \\
\max (i, j)<\max (p, q) .
\end{array}\right.
$$

(For example, the arc $V_{3}-V_{9}$ is less than the arc $V_{4}-V_{5}$.) Every partition of an $n$-gon has $n-3$ arcs which can be sorted from the smallest to the largest into an ordered sequence of arcs, i.e., each partition is associated with a unique ordered sequence of arcs. We define a partition $\boldsymbol{P}$ to be lexicographically less than a partition $Q$ if the ordered sequence of arcs associated with $P$ is lexicographically less than that associated with $Q$. 
When there is more than one optimum partition, we use the l-optimum partition (i.e., lexicographically-optimum partition) to mean the lexicographically smallest optimum partition, and use an optimum partition to mean some partition of minimum cost.

We shall use $V_{a}, V_{b}, \cdots$ to denote vertices which are unordered in weights, and $T_{i j k}$ to denote the product of the weights of any three vertices $V_{i}, V_{j}$ and $V_{k}$.

THEOREM 1. For every way of choosing $V_{1}, V_{2}, \cdots$ (as prescribed), there is always an optimum partition containing $V_{1}-V_{2}$ and $V_{1}-V_{3}$. (Here, $V_{1}-V_{2}$ and $V_{1}-V_{3}$ may be either arcs or sides.)

Proof. The proof is by induction, For the optimum partitions of a triangle and a 4-gon, the theorem is true. Assume that the theorem is true for all $k$-gons $(3 \leqq k \leqq n-1)$ and consider the optimum partitions of an $n$-gon.

From Lemma 3 , in any optimum partition, we can find at least two vertices having degree two. Call these two vertices $V_{i}$ and $V_{j}$. We can divide this into two cases.

(i) One of the two vertices $V_{i}$ (or $V_{j}$ ) is not $V_{1}, V_{2}$ or $V_{3}$ in some optimum partition of the $n$-gon. In this case, we can remove the vertex $V_{i}$ with its two sides and obtain an $(n-1)$-gon. In this $(n-1)$-gon, $V_{1}, V_{2}, V_{3}$ are the three vertices with smallest weights. By the induction assumption, $V_{1}$ is connected to both $V_{2}$ and $V_{3}$ in an optimum partition.

(ii) Consider the complementary case of (i), in all the optimum partitions of the $n$-gon, all the vertices with degree two are from the set $\left\{V_{1}, V_{2}, V_{3}\right\}$. (In this case, there will be at most three vertices with degree two in every optimum partition.) We have the following three subcases:

(a) $V_{i}=V_{2}$ and $V_{j}=V_{3}$ in some optimum partition of the $n$-gon, i.e., both $V_{2}$ and $V_{3}$ have degree two simultaneously. In this case, we first remove $V_{2}$ with its two sides and form an ( $n-1)$-gon. By the induction assumption, $V_{1}, V_{3}$ must be connected in some optimum partition. If $V_{1}-V_{3}$ appears as an arc, it reduces to (i). So $V_{1}-V_{3}$ must appear as a side of the $(n-1)$-gon, and reattaching $V_{2}$ to the $(n-1)$-gon shows that either $V_{1}, V_{2}$ and $V_{3}$ are mutually adjacent or $V_{1}-V_{3}$ is a side of the $n$-gon. In the former case, the proof is complete, so we assume that $V_{1}-V_{3}$ is a side of the $n$-gon. Similarly, we can remove $V_{3}$ with its two sides and show that $V_{1}, V_{2}$ are connected by a side of the $n$-gon.

(b) $V_{i}=V_{1}$ and $V_{j}=V_{2}$ in some optimum partition of the $n$-gon, i.e., $V_{1}$ and $V_{2}$ both have degree two simultaneously. In this case, we can first remove $V_{1}$ and form an $(n-1)$-gon where $V_{2}, V_{3}, V_{4}$ are the three vertices with smallest weights. By the induction assumption, $V_{2}$ is connected to both $V_{3}$ and $V_{4}$ in an optimum partition. If $V_{2}-V_{3}$ or $V_{2}-V_{4}$ appears as an arc, it reduces to (i). Hence, $V_{2}-V_{3}$ and $V_{2}-V_{4}$ must both be sides of the $n$-gon. Similarly, we can remove $V_{2}$ with its two sides and form an $(n-1)$-gon where $V_{1}, V_{3}, V_{4}$ are the three vertices with smallest weights. Again, $V_{1}$ must be connected to $V_{3}$ and $V_{4}$ by sides of the $n$-gon. But for any $n$-gon with $n \geqq 5$, it is impossible to have $V_{3}$ and $V_{4}$ both adjacent to $V_{1}$ and $V_{2}$ at the same time, i.e., $V_{1}$ and $V_{2}$ cannot both have degree two in an optimum partition of any $n$-gon with $n \geqq 5$.

(c) $V_{i}=V_{1}, V_{j}=V_{3}$ in some optimum partition of the $n$-gon. By argument similar to (b), we can show that $V_{2}$ must be adjacent to $V_{1}$ and $V_{3}$ in the $n$-gon. The situation is as shown in Fig. 3(a). Then the partition in Fig. 3(b) is cheaper because

$$
T_{123} \leq T_{12 q}
$$

and $C\left(w_{1}, w_{q}, w_{y}, w_{t}, w_{x}, w_{p}, w_{3}\right) \leqq C\left(w_{2}, w_{q}, w_{y}, w_{t}, w_{x}, w_{p}, w_{3}\right)$ according to Lemma 4. 


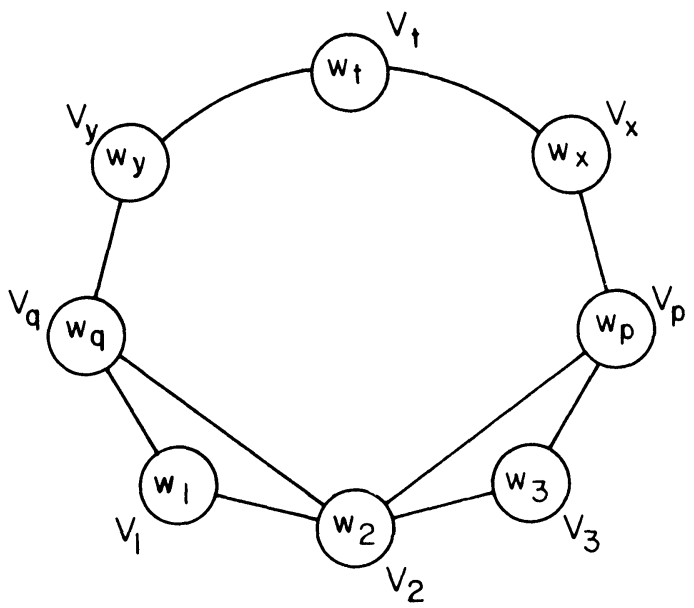

(a)

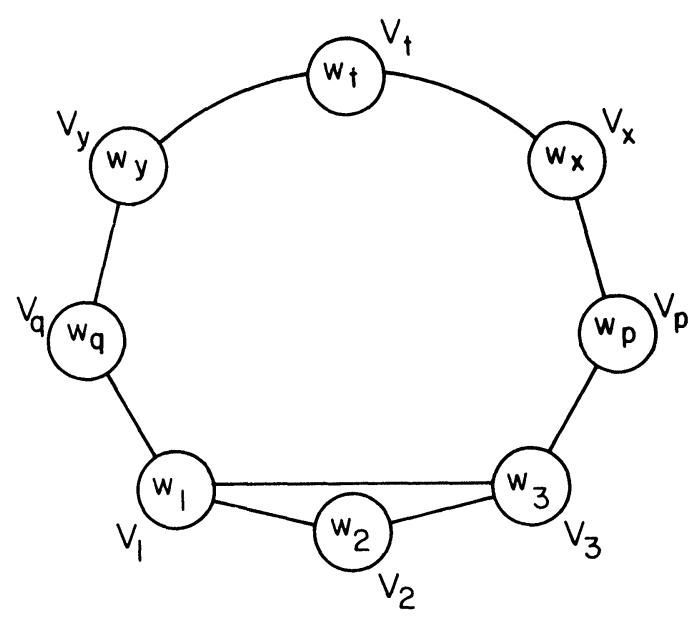

(b)

FIG. 3

COROllary 1. For every way of choosing $V_{1}, V_{2}, \cdots$ (as prescribed), the l-optimum partition always contains $V_{1}-V_{2}$ and $V_{1}-V_{3}$.

Proof. It follows from Theorem 1 and the definition of the $l$-optimum partition.

Once we know $V_{1}-V_{2}$ and $V_{1}-V_{3}$ always exist in the $l$-optimum partition, we can use this fact recursively. Hence, in finding the $l$-optimum partition of a given polygon, we can decompose it into subpolygons by joining the smallest vertex with the second smallest and third smallest vertices repeatedly, until each of these subpolygons has the property that its smallest vertex is adjacent to both its second smallest and third smallest vertices.

A polygon having $V_{1}$ adjacent to $V_{2}$ and $V_{3}$ by sides will be called a basic polygon.

THEOREM 2. A necessary but not sufficient condition for $V_{2}-V_{3}$ to exist in an optimum partition of a basic polygon is

$$
\frac{1}{w_{1}}+\frac{1}{w_{4}} \leqq \frac{1}{w_{2}}+\frac{1}{w_{3}}
$$

Furthermore, if $V_{2}-V_{3}$ is not present in the l-optimum partition, then $V_{1}, V_{4}$ are always connected in the l-optimum partition.

Proof. If $V_{2}, V_{3}$ are not connected in the $l$-optimum partition of a basic polygon, the degree of $V_{1}$ is greater than or equal to 3. Let $V_{p}$ be a vertex in the polygon and $V_{1}, V_{p}$ be connected in the $l$-optimum partition. $V_{4}$ is either in the subpolygon containing $V_{1}, V_{2}$ and $V_{p}$ or in the subpolygon containing $V_{1}, V_{3}$ and $V_{p}$. In either case, $V_{4}$ will be the third smallest vertex in the subpolygon. From Corollary $1, V_{1}, V_{4}$ are connected in the $l$-optimum partition of the subpolygon and it also follows that $V_{1}, V_{4}$ are connected in the $l$-optimum partition of the basic polygon.

If $V_{2}, V_{3}$ are connected in an optimum partition, then we have an $(n-1)$-gon where $V_{2}$ is the smallest vertex and $V_{4}$ is the third smallest vertex. By Theorem 1 , there exists an optimum partition of the $(n-1)$-gon in which $V_{2}, V_{4}$ are connected. Thus by induction on $n$, we can assume that $V_{4}$ is adjacent to $V_{2}$ in the basic polygon as shown in Fig. 4(a). 


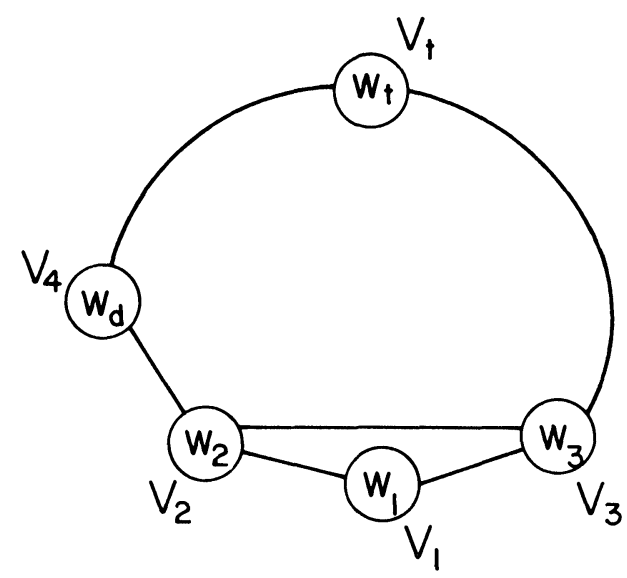

(a)

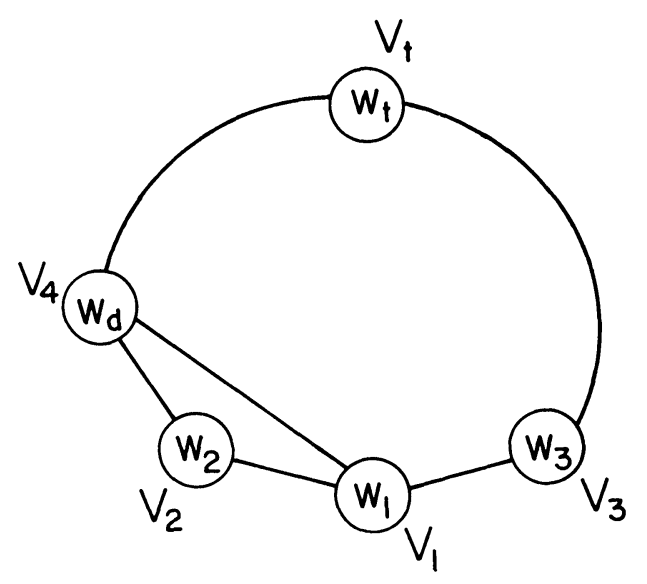

(b)

FIG. 4

The cost of the partition in Fig. 4(a) is

$$
T_{123}+C\left(w_{2}, w_{4}, \cdots, w_{t}, \cdots, w_{3}\right),
$$

and the cost of the partition in Fig. 4(b) is

$$
T_{124}+C\left(w_{1}, w_{4}, \cdots, w_{t}, \cdots, w_{3}\right)
$$

According to Lemma 4,

$$
C\left(w_{1}, w_{4}, \cdots, w_{t}, \cdots, w_{3}\right) \leqq C\left(w_{2}, w_{4}, \cdots, w_{t}, \cdots, w_{3}\right) .
$$

Since the weights of the vertices between $V_{4}$ and $V_{3}$ in the clockwise direction are all greater than or equal to $w_{4}$, the difference between the right-hand side and the left-hand side of (9) is at least

$$
T_{243}-T_{143} \text {. }
$$

So the necessary condition for (7) to be no greater than (8) is

$$
T_{123}+T_{243} \leqq T_{124}+T_{134}
$$

or

$$
\frac{1}{w_{1}}+\frac{1}{w_{4}} \leqq \frac{1}{w_{2}}+\frac{1}{w_{3}}
$$

LEMMA 5. In an optimum partition of an n-gon, let $V_{x}, V_{y}, V_{z}$ and $V_{w}$ be four vertices of an inscribed quadrilateral ( $V_{x}$ and $V_{z}$ are not adjacent in the quadrilateral). $A$ necessary condition for $V_{x}-V_{z}$ to exist is

$$
\frac{1}{w_{x}}+\frac{1}{w_{z}} \geqq \frac{1}{w_{y}}+\frac{1}{w_{w}} .
$$

Proof. The cost of partitioning the quadrilateral by the arc $V_{x}-V_{z}$ is

$$
T_{x y z}+T_{x z w},
$$

and the cost partitioning the quadrilateral by the arc $V_{y}-V_{w}$ is

$$
T_{x y w}+T_{y z w} \text {. }
$$

For optimality, we have $(11) \leqq(12)$ which is $(10)$. 
Note that if strict inequality holds in (10), the necessary condition is also sufficient. If equality holds in (10), the condition is sufficient for $V_{x}-V_{z}$ to exist in the $l$-optimum partition provided $\min (x, z)<\min (y, w)$. This lemma is a generalization of [3, Lemma 1] where $V_{y}$ is the vertex with the smallest weight and $V_{x}, V_{w}, V_{z}$ are three consecutive vertices with $w_{w}$ greater than both $w_{x}$ and $w_{z}$.

A partition is called stable if every quadrilateral in the partition satisfies (10).

Corollary 2. An optimum partition is stable but a stable partition may not be optimum.

Proof. The fact that an optimum partition has to be stable follows from Lemma 5. Figure 5 gives an example that a stable partition may not be optimum.

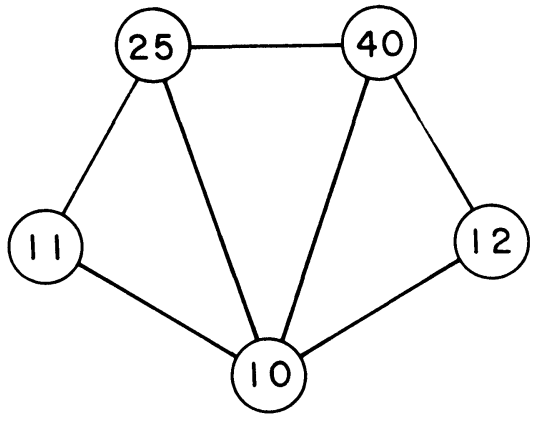

(a) a stable partition

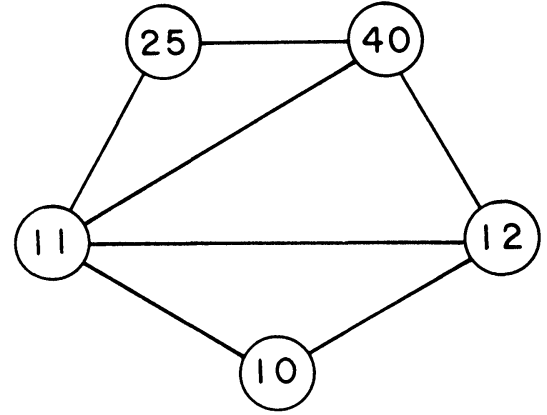

(b) the optimum partition

FIG. 5

In any partition of an $n$-gon, every arc dissects a unique quadrilateral. Let $V_{x}, V_{y}, V_{z}, V_{w}$ be the four vectices of an inscribed quadrilateral and $V_{x}-V_{z}$ be the arc which dissects the quadrilateral. We define $V_{x}-V_{z}$ to be a vertical arc if (13) or (14) is satisfied.

$$
\begin{gathered}
\min \left(w_{x}, w_{z}\right)<\min \left(w_{y}, w_{w}\right), \\
\min \left(w_{x}, w_{z}\right)=\min \left(w_{y}, w_{w}\right), \quad \max \left(w_{x}, w_{z}\right) \leqq \max \left(w_{y}, w_{w}\right) .
\end{gathered}
$$

We define $V_{x}-V_{z}$ to be a horizontal arc if (15) is satisfied

$$
\min \left(w_{x}, w_{z}\right)>\min \left(w_{y}, w_{w}\right), \quad \max \left(w_{x}, w_{z}\right)<\max \left(w_{y}, w_{w}\right) .
$$

For brevity, we shall use $h$-arcs and $v$-arcs to denote horizontal arcs and vertical arcs from now on.

COROllary 3. All arcs in an optimum partition must be either vertical arcs or horizontal arcs.

Proof. Let $V_{x}-V_{z}$ be an arc which is neither vertical nor horizontal. There are two cases:

Case 1. $\min \left(w_{x}, w_{z}\right)=\min \left(w_{y}, w_{w}\right)$ and $\max \left(w_{x}, w_{z}\right)>\max \left(w_{y}, w_{w}\right)$;

Case 2. $\min \left(w_{x}, w_{z}\right)>\min \left(w_{y}, w_{w}\right)$ and $\max \left(w_{x}, w_{z}\right) \geqq \max \left(w_{y}, w_{w}\right)$.

In both cases, the inequality (10) in Lemma 5 cannot be satisfied. This implies that the partition is not stable and hence cannot be optimum.

THEOREM 3. Let $V_{x}$ and $V_{z}$ be two arbitrary vertices which are not adjacent in a polygon, and $V_{w}$ be the smallest vertex from $V_{x}$ to $V_{z}$ in the clockwise manner $\left(V_{w} \neq\right.$ $\left.V_{x}, V_{w} \neq V_{z}\right)$, and $V_{y}$ be the smallest vertex from $V_{z}$ to $V_{x}$ in the clockwise manner 


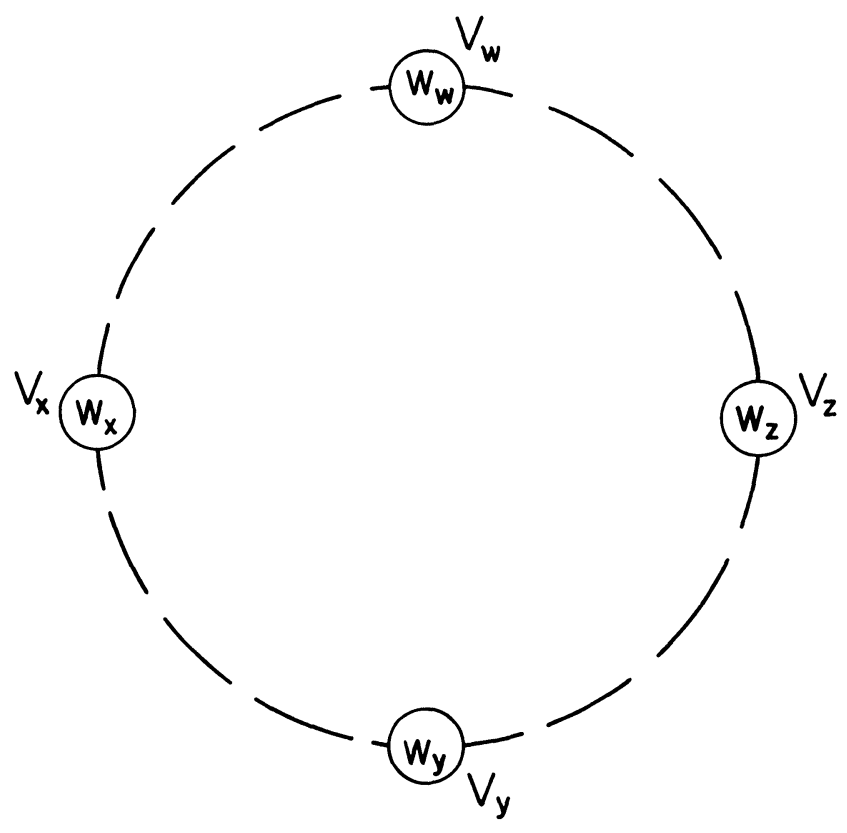

FiG. 6

$\left(V_{y} \neq V_{x}, V_{y} \neq V_{z}\right)$. This is shown in Fig. 6, where without loss of generality we assume that $V_{x}<V_{z}$ and $V_{y}<V_{w}$. A necessary condition for $V_{x}-V_{z}$ to exist as an h-arc in the l-optimum partition is that

$$
w_{y}<w_{x} \leqq w_{z}<w_{w}
$$

(Note that the necessary condition still holds when the positions of $V_{y}$ and $V_{w}$ are interchanged.)

Proof. The proof is by contradiction. If $w_{x} \leqq w_{y}, w_{x}$ must be equal to the smallest weight $w_{1}$, and $V_{x}-V_{z}$ can never satisfy (15). Hence, in order that $V_{x}-V_{z}$ exist as an $h$-arc in the $l$-optimum partition, we must have $w_{y}<w_{x} \leqq w_{z}$. Since $V_{y}$ is the smallest vertex from $V_{z}$ to $V_{x}$ in the clockwise manner and $V_{x}<V_{w}$, we must have $V_{y}=V_{1}$.

Assume for the moment that $V_{3}<V_{x}<V_{z}$. From Corollary 1 , both $V_{1}-V_{2}$ and $V_{1}-V_{3}$ exist in the $l$-optimum partition, and the two arcs would divide the polygon into subpolygons. If $V_{x}$ and $V_{z}$ are in different subpolygons, then they cannot be connected in the $l$-optimum partition. Without loss of generality, we can assume that the polygon is a basic polygon. In this basic polygon, either $V_{2}-V_{3}$ or $V_{1}-V_{4}$ exists in the $l$-optimum partition (Theorem 2).

If $V_{2}, V_{3}$ are connected, then $V_{x}$ and $V_{z}$ are both in a smaller polygon in which we can treat $V_{2}$ as the smallest vertex and repeat the argument. If $V_{1}, V_{4}$ are connected, the basic polygon is again divided into two subpolygons and $V_{x}$ and $V_{z}$ both have to be in one of the subpolygons and the subpolygon has at most $n-1$ sides. (Otherwise $V_{x}-V_{z}$ can never exist in the $l$-optimum partition.) The successive reduction in the size of the polygon will either make the connection $V_{x}-V_{z}$ impossible, or force $V_{x}$ and $V_{z}$ to become the second smallest and the third smallest vertices in a basic subpolygon. Let $V_{m}$ be the smallest vertex in this basic subpolygon. In order that $V_{x}-V_{z}$ appear as an $h$-arc, we must have $w_{x}>w_{m}$. From Theorem 2, the necessary condition for $V_{x}-V_{z}$ (i.e., $V_{2}-V_{3}$ ) to exist in an optimum partition of the subpolygon 
is

$$
\frac{1}{w_{x}}+\frac{1}{w_{z}} \geqq \frac{1}{w_{m}}+\frac{1}{w_{w}}
$$

Since $w_{x}>w_{m}$, the inequality is valid only if $w_{z}<w_{w}$.

Corollary 4. A weaker necessary condition for $V_{x}-V_{z}$ to exist as an $h$-arc in the l-optimum partition is that

$$
V_{y}<V_{x}<V_{z}<V_{w}
$$

Proof. This follows from Theorem 3.

We call any arc which satisfies this weaker necessary condition a potential $h$-arc. Let $P$ be the set of potential $h$-arcs in the $n$-gon and $H$ be the set of $h$-arcs in the $l$-optimum partition, we have $P \supseteq H$ where the inclusion could be proper.

COROllaRY 5. Let $V_{w}$ be the largest vertex in the polygon and $V_{x}$ and $V_{z}$ be its two neighboring vertices. If there exists a vertex $V_{y}$ such that $V_{y}<V_{x}$ and $V_{y}<V_{z}$, then $V_{x}-V_{z}$ is a potential h-arc.

Proof. This follows directly from Corollary 4 where there is only one vertex between $V_{x}$ and $V_{z}$.

Two arcs are called compatible if both arcs can exist simultaneously in a partition. Assume that all weights of the vertices are distinct, then there are $(n-1)$ ! distinct permutations of the weights around an $n$-gon. For example, the weights $10,11,25$, 40,12 in Fig. 5(a) correspond to the permutation $w_{1}, w_{2}, w_{4}, w_{5}, w_{3}$ (where $w_{1}<w_{2}<$ $w_{3}<w_{4}<w_{5}$ ). There are infinitely many values of weights which correspond to the same permutation. For example, 1, 16, 34, 77, 29 also corresponds to $w_{1}, w_{2}, w_{4}, w_{5}, w_{3}$ but its optimum partition is different from that of $10,11,25,40,12$. However, all the potential $h$-arcs in all the $n$-gons with the same permutation of weights are compatible. We state this remarkable fact as Theorem 4.

THEOREM 4. All potential h-arcs are compatible.

Proof. The proof is by contradiction. Let $V_{x}, V_{y}, V_{z}$ and $V_{w}$ be the four vertices described in Theorem 3. Hence, we have $V_{y}<V_{x}<V_{z}<V_{w}$ and $V_{x}-V_{z}$ is a potential $h$-arc. Let $V_{p}-V_{q}$ be a potential $h$-arc which is not compatible to $V_{x}-V_{z}$, as shown in Fig. 7. Without loss of generality, we can assume $V_{p}<V_{q}$. (The proof for the case $V_{q}<V_{p}$ is similar to that which follows.)

Since $V_{w}$ is the smallest vertex between $V_{x}$ and $V_{z}$ in the clockwise manner, we have $V_{z}<V_{w}<V_{q}$. Hence, we have either $V_{y}<V_{p}<V_{z}<V_{q}$ or $V_{y}<V_{z}<V_{p}<V_{q}$. Both cases violate Corollary 4 and $V_{p}-V_{q}$ cannot be a potential $h$-arc.

Note that the potential $h$-arc $V_{x}-V_{z}$ always dissects the $n$-gon into two subpolygons and one of these subpolygons has the property that all its vertices except $V_{x}$ and $V_{z}$ have weights no smaller than $\max \left(w_{x}, w_{z}\right)$. We shall call this subpolygon the upper subpolygon of $V_{x}-V_{z}$. For example, the subpolygon $V_{x}-\cdots-V_{w}-\cdots-V_{z}$ in Fig. 7 is the upper subpolygon of $V_{x}-V_{z}$.

Using Corollary 4 and Theorem 4 , we can generate all the potential $h$-arcs of a polygon.

Let $V_{x}-V_{z}$ be the arc defined in Corollary 5, i.e., $V_{1}<V_{x}<V_{z}<V_{w}$. The arc $V_{x}-V_{z}$ is a potential $h$-arc compatible with all other potential $h$-arcs in the $n$-gon. Furthermore, there is no other potential $h$-arc in its upper subpolygon. Now consider the $(n-1)$-gon obtained by cutting out $V_{w}$. In this $(n-1)$-gon, let $V_{w^{\prime}}$ be the largest vertex and $V_{x^{\prime}}$ and $V_{z^{\prime}}$ be the two neighbors of $V_{w^{\prime}}$ where $V_{1}<V_{x^{\prime}}<V_{z^{\prime}}<V_{w^{\prime}}$. Then $V_{x^{\prime}}-V_{z^{\prime}}$ is again a potential $h$-arc compatible with all other potential $h$-arcs in the 


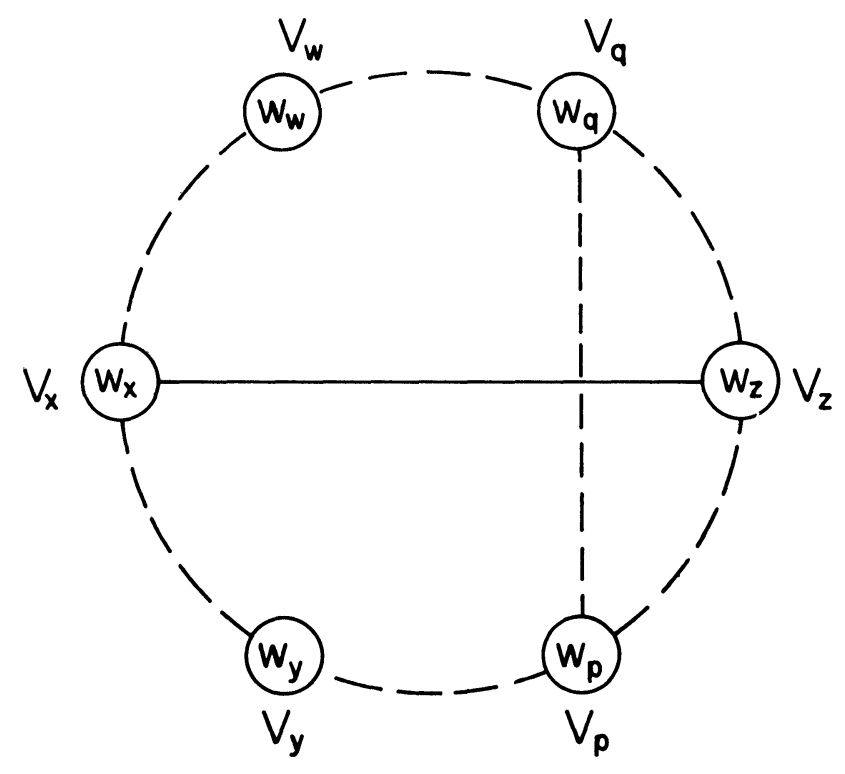

FIG. 7

$n$-gon and there is no other potential $h$-arc in its upper subpolygon which has not been generated. This is true even if $V_{w}$ is in the upper subpolygon of $V_{x^{\prime}}-V_{z^{\prime}}$. If we repeat the process of cutting out the largest vertex, we get a set $P$ of arcs, all of which satisfy Corollary 4 . The $h$-arcs of the $l$-optimum partition must be a subset of these arcs.

The process of cutting out the largest vertex can be made into an algorithm which is $O(n)$. We shall call this algorithm the one-sweep algorithm. The output of the one-sweep algorithm is a set $S$ of $n-3$ arcs. $S$ is empty initially.

The one-sweep algorithm. Starting from the smallest vertex, say $V_{1}$, we travel in the clockwise direction around the polygon and push the weights of the vertices successively onto the stack as follows ( $w_{1}$ will be at the bottom of the stack).

(a) Let $V_{t}$ be the top element on the stack, $V_{t-1}$ be the element immediately below $V_{t}$, and $V_{c}$ be the element to be pushed onto the stack. If there are two or more vertices on the stack and $w_{t}>w_{c}$, add $V_{t-1}-V_{c}$ to $S$, pop $V_{t}$ off the stack; if there is only one vertex on the stack or $w_{t} \leqq w_{c}$, push $w_{c}$ onto the stack. Repeat this step until the $n$th vertex has been pushed onto the stack.

(b) If there are more than three vertices on the stack, add $V_{t-1}-V_{1}$ to $S$, pop $V_{t}$ off the stack and repeat this step, else stop.

Since we do not check for the existence of a smallest vertex whose weight is no larger than those of the two neighbors of the largest vertex, i.e., the existence of the vertex $V_{y}$ in Corollary 4, not all the $n-3$ arcs generated by the algorithm are potential $h$-arcs. However, it is not difficult to verify that the one-sweep algorithm always generates a set $S$ of $n-3$ arcs which contains the set $P$ of all potential $h$-arcs which contains the set $H$ of all $h$-arcs in the $l$-optimum partition of the $n$-gon, i.e.,

$$
S \supseteq P \supseteq H,
$$

where each inclusion could be proper. For example, if the weights of the vertices around the $n$-gon in the clockwise direction are $w_{1}, w_{2}, \cdots, w_{n}$ where $w_{1} \leqq w_{2} \leqq \cdots \leqq$ $w_{n}$, none of the arcs in the $n$-gon can satisfy Corollary 4 and hence there are no 
potential $h$-arcs in the $n$-gon. The one-sweep algorithm would still generate $n-3$ arcs for the $n$-gon but none of the arcs generated is a potential $h$-arc.

3. Conclusion. In this paper, we have presented several theorems on the polygon partitioning problem. Some of these theorems are characterizations of the optimum partitions of any $n$-sided convex polygon, while the others apply to the unique lexicographically smallest optimum partition. Based on these theorems an $O(n)$ algorithm for finding a near-optimum partition can be developed [12]. The cost of the partition produced by the heuristic algorithm never exceeds $1.155 C_{\text {opt }}$, where $C_{\text {opt }}$ is the optimum cost of partitioning the polygon. An $O(n \log n)$ algorithm for finding the unique lexicographically smallest optimum partition will be presented in Part II [13].

Acknowledgment. The authors would like to thank the referees for their helpful comments in revising the manuscript.

\section{REFERENCES}

[1] A. V. Aho, J. E. Hopcroft And J. D. Ullman, The Design and Analysis of Computer Algorithms, Addison-Wesley, Reading, MA, 1974.

[2] A. K. ChANDRA, Computing matrix chain product in near optimum time, IBM Res. Rep. RC5626 (\# 24393), IBM Thomas J. Watson Research Center, Yorktown Heights, NY, 1975.

[3] F. Y. CHIN, $A n O(n)$ algorithm for determining a near optimal computation order of matrix chain product, Comm. ACM, 21, (1978), pp. 544-549.

[4] L. E. Deimel, JR. ANd T. A. LAMPE, An invariance theorem concerning optimal computation of matrix chain products, Rep. TR79-14, North Carolina State Univ., Raleigh.

[5] G. A. Dirac, On rigid circuit graphs, Abh. Math. Sem., Univ. Hamburg, 25 (1961), pp. 71-76.

[6] M. GARDNer, Catalan numbers, Scientific American, June 1976, pp. 120-124.

[7] S. S. GodBOLE, An efficient computation of matrix chain products, IEEE Trans. Comput., C-22, (1973), pp. 864-866.

[8] H. W. Gould, Bell and Catalan numbers, Combinatorial Research Institute, Morgantown, WV., June 1977.

[9] T. C. Hu AND M. T. ShING, Computation of Matrix Chain Product, Abstract, Amer. Math. Soc., Vol. 1, (1980), p. 336.

[10] - Some theorems about matrix multiplications, Proc. 21st Annual IEEE Symposium on the Foundations of Computer Science, October 1980, pp. 28-35.

[11] Computation of matrix chain products, Proc. 1981 Army Numerical Analysis and Computations Conference, August 1981, pp. 615-628.

[12] — (1981), pp. 122-138.

[13] - Computation of matrix chain products. Part II, submitted. 